\title{
Basic design and simulation of a SPECT microscope for in vivo stem cell imaging
}

\author{
Rex A. Moats*a, Yang Tang ${ }^{\mathrm{a}}$, James W. Hugg ${ }^{\mathrm{b}}$, Dirk Meier ${ }^{\mathrm{c}}$, David Koos ${ }^{\mathrm{d}}$, Neal E. Hartsough ${ }^{\mathrm{e}}$, \\ Bradley E. Patt ${ }^{\mathrm{b}}$, Douglas J. Wagenaar ${ }^{\mathrm{b}}$ \\ ${ }^{a}$ Department of Radiology, Children's Hospital of Los Angeles, Los Angeles, CA, USA; \\ ${ }^{\mathrm{b}}$ Gamma Medica, Northridge, CA, USA; ' Gamma Medica, Oslo, Norway; \\ ${ }^{\mathrm{d}}$ California Institute of Technology, Pasadena, CA, USA; ${ }^{\mathrm{e}}$ DxRay, Northridge, California USA
}

\begin{abstract}
The need to understand the behavior of individual stem cells at the various stages of their differentiation and to assess the resulting reparative action in pre-clinical model systems, which typically involves laboratory animals, provides the motivation for imaging of stem cells in vivo at high resolution. Our initial focus is to image cells and cellular events at single cell resolution in vivo in shallow tissues (few $\mathrm{mm}$ of intervening tissue) in laboratory mice and rates. In order to accomplish this goal we are building a SPECT-based microscope. We based our design on earlier theoretical work with near-field coded apertures and have adjusted the components of the system to meet the real-world demands of instrument construction and of animal imaging. Our instrumental design possesses a reasonable trade-off between field-of-view, sensitivity, and contrast performance (photon penetration). A layered gold aperture containing 100 pinholes and intended for use in coded aperture imaging application has been designed and constructed. A silicon detector connected to a TimePix readout from the CERN collaborative group was selected for use in our prototype microscope because of its ultra-high spatial and energy resolution capabilities. The combination of the source, aperture, and detector has been modeled and the coded aperture reconstruction of simulated sources is presented in this work.
\end{abstract}

Keywords: nuclear medicine, microscope, stem cells, imaging, coded aperture, silicon detector, simulation

\section{INTRODUCTION}

Radio-labeled blood cells have been used in clinical nuclear medicine since the 1970s. The procedure involves the extraction of the patient's blood, labeling the cells, and then re-injection of the blood cells back into the patient to look for internal bleeding or vasculature (red blood cells) or sites of infection (white blood cells). This re-injection technique limits the imaging time window due to the half-life of the radioisotope. New techniques for nuclear imaging of cells have been developed. Especially relevant to our quest is the production of genetically-altered stem cells such as those developed by our collaborators Gazit and Pelled of Cedars-Sinai Medical Center which upon differentiation express the human sodium iodide symporter ${ }^{1}$. Their cell labeling technique allows "free" radioactive technetium or iodine atoms to

Medical Imaging 2011: Physics of Medical Imaging, edited by Norbert J. Pelc, Ehsan Samei, Robert M. Nishikawa, Proc. of SPIE Vol. 7961, 79614B - (C) 2011 SPIE · CCC code: 1605-7422/11/\$18 - doi: 10.1117/12.878427 
be taken up by the symporter-expressing cells, thereby allowing them to be imaged within biological appropriate time windows and, when desired, only after differentiation. The differentiated stem cell is the class currently expected to be responsible for many of the proposed curative effects of stem cell therapies.

Imaging the localized and migratory behavior of stem cells in vivo is difficult because of their small size $(\sim 20 \mu \mathrm{m})$ and the thickness of tissue intervening between the cell and the imaging detector. Visible light photons are used to view fluorescently labeled cells through special fiber optics that are inserted into the near vicinity (up to $300 \mu \mathrm{m}$ ) of the cells. Longer range microscopy is not currently possible with visible light due to limitations imposed by photon scatter. We propose to circumvent some of these difficulties by imaging individual cells in vivo using radioactive labeling with lowenergy photon emitting radioisotopes. Following the theoretical foundation of the work of others in the field we are implementing a pragmatic design for high resolution nuclear imaging at low energies and this manuscript details our progress to date.

\section{NUCLEAR MICROSCOPY}

\subsection{Low energies}

Gamma-ray and x-ray photons associated with radioactive decay can have energies below $10 \mathrm{keV}$ (e.g., 5.9 and $6.5 \mathrm{keV}$ from ${ }^{55} \mathrm{Fe}$ ). Commonly used isotopes ${ }^{57} \mathrm{Co},{ }^{99 \mathrm{~m}} \mathrm{Tc},{ }^{109} \mathrm{Cd}$, and ${ }^{111} \mathrm{In}$ have low energy emissions: $14 \mathrm{keV}$ gamma; $18,20.6$ $\mathrm{keV}$ x-rays; 22, $25 \mathrm{keV}$ x-rays; and 23, $26 \mathrm{keV}$ x-rays, respectively. The photons are emitted from the radioactive atom itself, so theoretically cellular and even sub-cellular resolution would be possible. Our method is based on a form of coded aperture imaging. A single pinhole can achieve high resolution but with low sensitivity. Because of the low energies, relatively thin heavy metal (e.g., Au, W, Pt) foils can be used, with thicknesses typically in the 50-200 $\mu \mathrm{m}$ range. Because we hope to achieve resolution $<50 \mu \mathrm{m}$, the aspect ratio of the holes must be relatively large, especially for energies approaching $20 \mathrm{keV}$, so we designed conical-shaped holes to avoid limited FOV and the fabrication challenges of high aspect ratios.

\subsection{Coded apertures}

An overview of coded aperture imaging is given by Skinner ${ }^{2}$, and a low-energy, near-field application with ${ }^{55} \mathrm{Fe}$ was reported by Accorsi ${ }^{3}$, achieving $20 \mu \mathrm{m}$ resolution. A modified uniformly redundant array (MURA) was designed by Dr. Accorsi for our use in this project (Figure 1).

\subsection{Detector}

A $300 \mu \mathrm{m}$-thick silicon detector attached to the "TimePix"4 readout was selected due to its pixel pitch and energy resolving capability (Figure 2). TimePix is a version of the MediPix2 ASIC, made available for this project by the Medipix2 Collaboration under the direction of Dr. Michael Campbell (CERN) and Dr. Michael Fiederle (University of Freiburg).

Higher sensitivity can be achieved by using multiple Si/TimePix detectors (for expanded area) and by replacing the silicon with CdTe after feasibility is shown. Figure 3 shows pixellated images of counts (two left figures) from ${ }^{55} \mathrm{Fe}$ and 
${ }^{57} \mathrm{Co}$. At middle right of Figure 3 the two spectra are clearly separated, with an ROC analysis showing the separation of the two peaks at the far right.

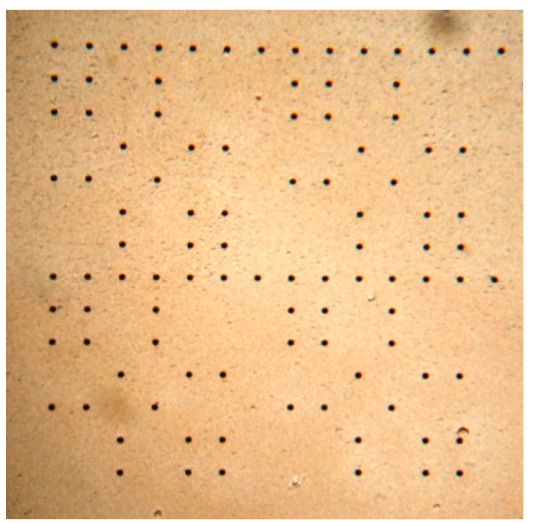

Figure 1. The MURA coded aperture pattern of holes in Au foil. A few incidental surface artifacts are present but not significant. A 25 whole pattern was repeated $4 \mathrm{X}$. Originally the design called for closer packing of the pinholes but in order to use the chosen method of mask construction a looser configuration of the holes had to be allowed for the prototype instrument.

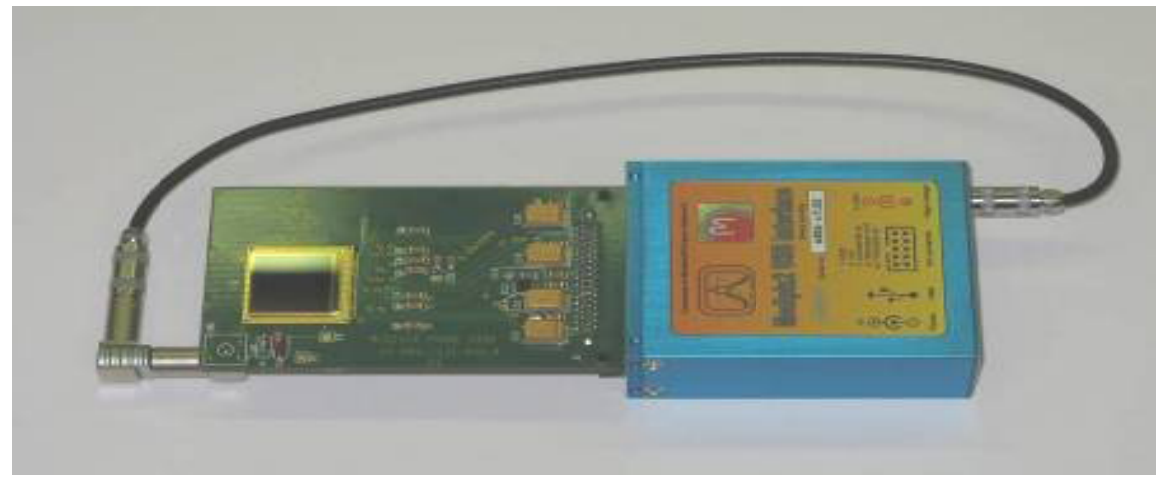

Figure 2. The $55 \mu \mathrm{m}$-pitch, 256x256 "TimePix" readout with $300 \mu \mathrm{m}$ thick Si detector attached, selected for use in our nuclear microscope.
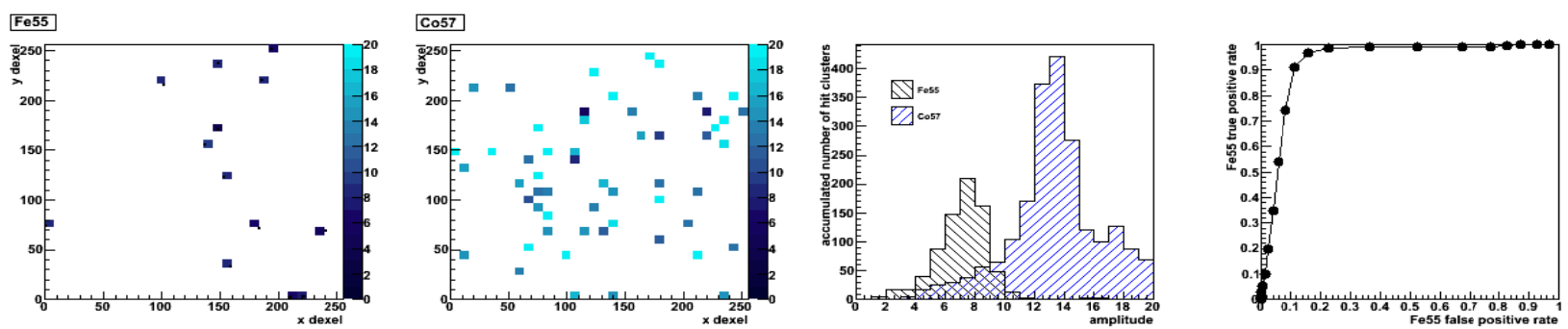

Figure 3. Energy discrimination capability of Si/TimePix detector, using $55 \mathrm{Fe}(6 \mathrm{keV})$ and $57 \mathrm{Co}(14 \mathrm{keV})$. Note that the high energy photons (122 and $136 \mathrm{keV}$ ) from ${ }^{57} \mathrm{Co}$ do not add significantly to the background of the spectrum. 


\section{CODED APERTURES IN MICROSCOPY}

\subsection{Other Nuclear Microscope Research}

Miller at the University of Arizona has employed an image intensifier detector known as the "bazooka" due to its initially long focal length. Meng at the University of Illinois ${ }^{6}$ and Accorsi at Children's Hospital, Philadelphia ${ }^{7}$ are also doing microscope research.

\subsection{Microscope design}

Figure 4 shows a schematic depiction of our microscope, which depends upon use of magnification to achieve high resolution.

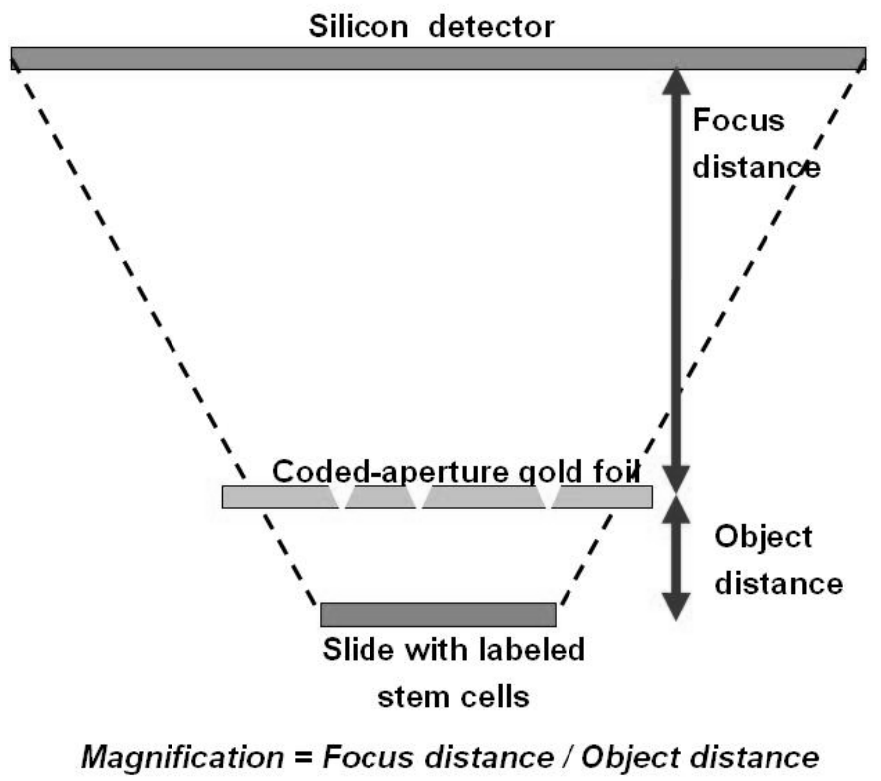

Figure 4. Schematic diagram of microscope geometry. The extended area of the detector will be achieved by using relative motion of the source/aperture and the $\mathrm{Si} / \mathrm{TimePix}$ detector.

\section{CODED APERTURE FABRICATION}

\subsection{Trade-offs}

Small holes, of the same order of the resolution desired, must be made in a foil sufficiently thick to stop the gamma-rays or x-rays. A large aspect ratio limits the field-of-view, so we settle for a conical shape as shown in Figure 5.

\subsection{Layered Approach}

We used two layers of gold foil with different-sized cylindrical holes to approximate the desired conical hole shapes shown in Figure 5a. A schematic of the concept is shown on the right side of Figure 5a and a photograph of one aperature hole of the manufactured foil is shown in Figure 5b. 


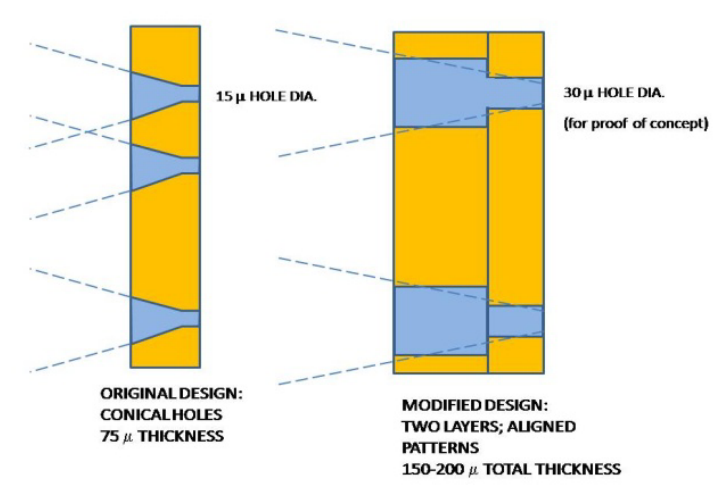

Figure 5a. Use of layered gold foils (right) to emulate the desired conical hole shapes (left).

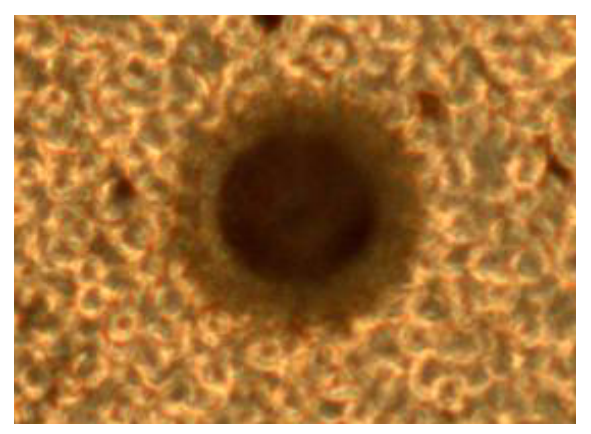

Figure 5b. Photograph of one of the coded aperture holes, showing a $30 \mu \mathrm{m}$ diameter hole in $60 \mu \mathrm{m}$ thick Au foil aligned with a 145 $\mu \mathrm{m}$ hole in $90 \mu \mathrm{m}$ thick Au foil.

\section{INSTRUMENT DESIGN}

The prototype instrument is designed to go onto an optical table with the standard post/rail type frame onto which other components can be attached. The detector is attached to the rail in a fixed position while the mask and sample (mouse) are on X-Y-Z stages as shown in Figure 6. The detector is pinned in place to avoid any vibration in the detector assembly itself. The stages are capable of sub-micron resolution in X-Y-Z. The stages holding the sample are designed to be strong enough and of similar materials to mimimize differential expansion on small temperature changes. But a covering box with climate controls (not shown) is required to minimize any temperature changes and eventually to provide support for animal experiments. Anti-coincidence shielding to remove cosmic-ray background may be required and remains to be designed if background rates prove to be excessive.

The instrument has some unique characterists: The size of the microscope turns out to be on the order of magnitude of the size of a mouse. The instrument is designed to allow the mask to object distance and thus the magnification to be variable. The range of field of view in the current configuration is only a few millimeters and will require that the location of the objects of interest are known ahead of time. This is the case in our planned stem cell experiments.

\section{CODED APERTURE SIMULATION}

\subsection{Theory}

Accorsi $^{3}$ describes the use of corelation integrals to deconvolve the multiplexed data that the radioactivity distribution creates on the detector through the MURA coded aperture. Optical corrections are made to transform parallel ray equations into the diverging (magnified) geometry used in our microscope. 


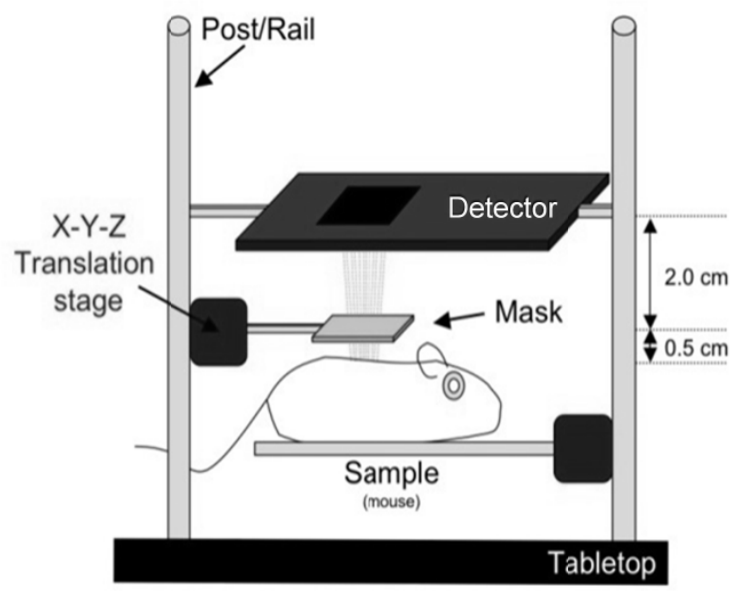

Figure 6. Schematic drawing of the sample/animal imaging system designed for use with the stationary Si/TimePix detector (shown at top). An X-Y-Z translational stage is capable of moving the coded aperture mask, while another stage moves the sample platform.

\subsection{Simulation Tool}

A simulation tool was created in MATLAB (Figure 7) based on Accorsi's work ${ }^{8}$, allowing the geometric parameters of the microscope design to be altered with graphical user interfaces.

At right is shown the resulting reconstruction of two point sources of cell-size through the 100-hole (4x25) MURA coded aperture of Figure 1. Note the residual artifacts of the coded aperture pattern from the reconstruction processes.

In the projection, each ray omitted from the object is geometrically traced, through the mask and projected to the detector plane. A constant PSF (Point Spread Function) is assumed over the detector. A 2D Gaussian kernel is convolved to the projection data to simulate the intrinsic PSF blurring. The geometric resolution is determined by the pinhole size and magnification.

The projected images are reconstructed in the frequency domain using the Fourier transform. The reconstruction decodes the patterned projected image into a reconstructed image by the delta decoding method ${ }^{8}$.

\section{SIMULATION RESULTS}

Figure 7 shows two simulated cell-sized point sources (marked in white circles) and their reconstructed image (at right) with uncorrected shadow artifacts from the coded aperture pattern. The simulation parameters for object, mask and detector in this simulation are shown in the panels of Figure 7. In the projection, the PSF blurring, Poisson noise and incidence angles are taken into account. 
The simulation showed that cell-like sources could be reconstructed even if they did not illuminate all 4 sets of holes of the MURA pattern. This opens the FOV wider than we originally expected.

It was noted that the near-field geometry allows resolution in depth to be found, as described by Accorsi ${ }^{7}$.

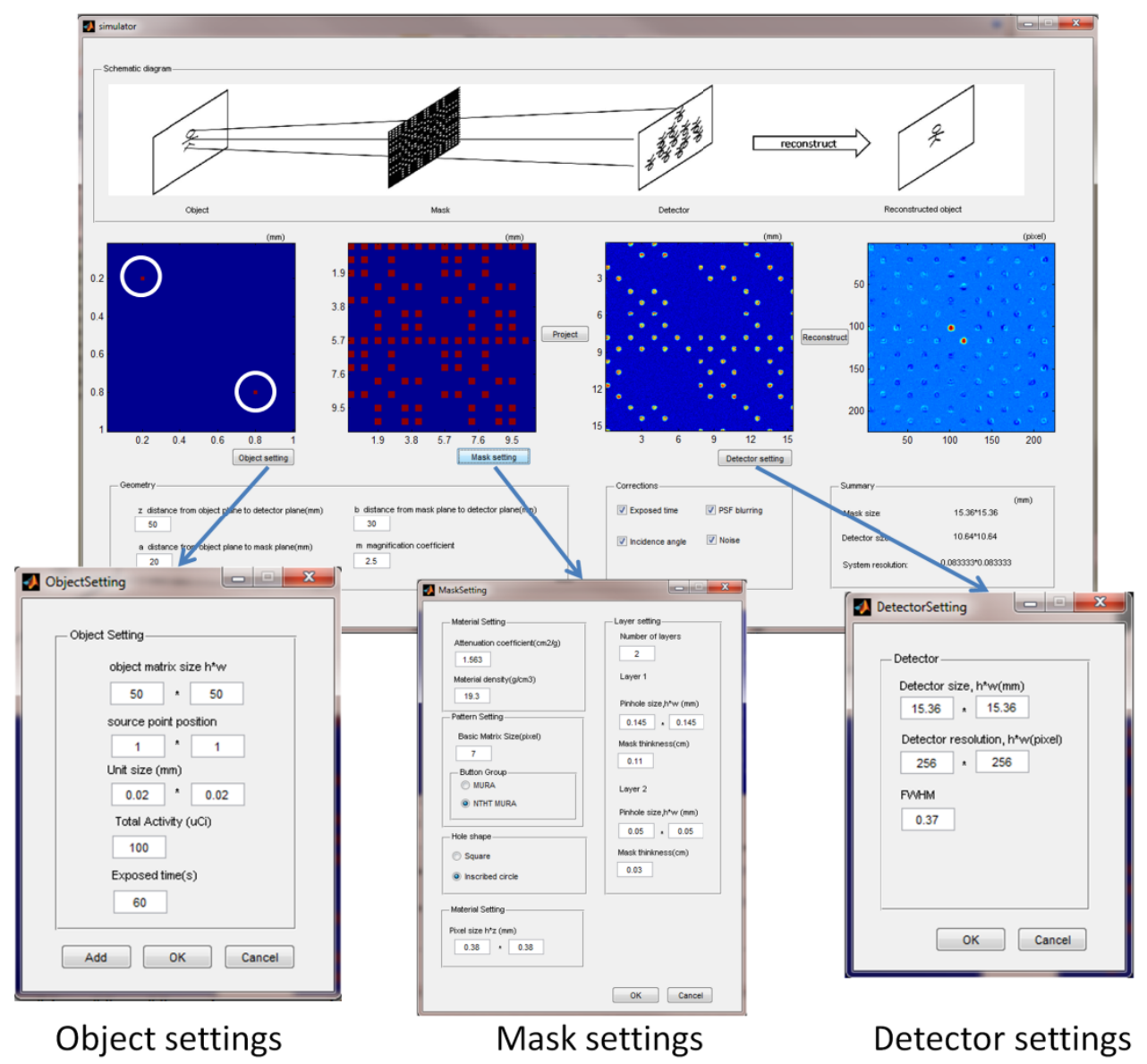

Figure 7. Graphical user interface created for the simulation of cell-sized sources, coded aperture patterns, and pixellated detectors. The relative placement of the source, coded aperture, and detector - as well as the size of the FOV and the extent of the hole pattern were adjustable parameters.

\section{CONCLUSION}

The low-energy photon emissions of radioisotopes labeled to stem cells can be used with coded apertures and highresolution pixellated, energy-discriminating detectors to achieve spatial resolution approaching the cellular range (as low as $20-30 \mu \mathrm{m})$. 


\section{ACKNOWLEDGEMENT}

We would like to thank Dan Gazit, Gadi Pelled, Todd Peterson, Gunnar Maehlum, William Barber, and Roberto Accorsi. Funding from the California Institute for Regenerative Medicine (CIRM), Tools \& Technologies I program, grant RT01-01027 is gratefully acknowledged.

\section{REFERENCES}

[1] Pelled, G., Ben Arav, A., Hock, C., Reynolds, D.G., Yazici, C., Zilberman, Y., Gazit, Z., Awwad, H., Gazit, D., Schwarz, E.M., "Direct Gene Therapy for Bone Regeneration: Gene Delivery, Animal Models, and Outcome Measures ," Tissue Engineering Part B: Reviews. 16(1), 13-20 (2010) .

[2] Skinner, G.K., "Imaging with coded aperture masks," Nucl Instrum Meth Phys Res, 221, 33-40(1984).

[3] Accorsi, R., " 20-mm resolution imaging of soft x-ray emitters," IEEE Tran Nucl Sci. 55(3), 889-897(2008).

[4] Llopart, X., Ballabriga, R., Campbell, M., Tlustos, L., Wong, W., "Timepix, a 65k programmable pixel readout chip for arrival time, energy and/or photon counting measurements," NIM A. 581, 485-494(2007).

[5] Miller, B.W., Barrett, H.H., Furenlid, L.R., Barber, B., Hunter, R.J., "Recent advances in BazookaSPECT: Realtime data processing of a g-ray microscope," Nuclear Instrum Methods A. 591(1),272-275(2008).

[6] Meng, L.J., Clinthorne, N.H., Skinner, S., Hay, R.V., Gross, M., "Design and feasibility study of a single photon emission microscope system for small animal I-125 imaging," IEEE Tran Nucl Sci. 53(3), 1168-1178(2006).

[7] Accorsi,R., "Analytic derivation of the longitudinal component of the three-dimensional point-spread function in coded-aperture laminography," Applied Optics. 44(28), 5872-5883(2005).

[8] Accorsi, R., "Design of near-field coded aperture cameras for high resolution medical and industrial gamma ray imaging," Ph.D Dissert MIT, (2001).

Proc. of SPIE Vol. 7961 79614B-8 